\title{
Automated Explanation of Research Informed Consent by Virtual Agents
}

\author{
Timothy Bickmore, ${ }^{1}$ Dina Utami, ${ }^{1}$ Shuo Zhou, ${ }^{1}$ Candace Sidner, ${ }^{2}$ Lisa Quintiliani, ${ }^{3}$ \\ and Michael Paasche-Orlow ${ }^{3}$ \\ ${ }^{1}$ College of Computer and Information Science, Northeastern University, Boston, MA \\ ${ }^{2}$ Worcester Polytechnic Institute, Worcester, MA \\ ${ }^{3}$ Boston Medical Center, Boston, MA \\ bickmore@ccs.neu.edu
}

\begin{abstract}
A virtual agent that explains research informed consent documents to study volunteers is described, along with a series of development efforts and evaluation studies. A study of nurse administration of informed consent finds that human explanations follow the structure of the document, and that much of information provided verbally is not contained in the document at all. A study of pedagogical strategies used by a virtual consent agent finds that automatic tailoring of document content based on users' knowledge receives the highest ratings of satisfaction compared to two control conditions that provided fixed amounts of information. We finally report on an approach that lets clinicians construct their own virtual agents for informed consent, along with a study that finds that nurses are able to use the system to develop and extend agents to explain their own study consent forms.
\end{abstract}

Keywords: Relational agent, embodied conversational agent, health literacy, medical informatics, health informatics.

\section{Introduction}

Informed Consent is an obligatory procedure in the US, in which a significant amount of technical and legal information is supposed to be taught to a layperson before they can agree to participate in a research study or clinical trial. It is a cornerstone in the ethical treatment of human subjects, the result of decades of debate about how to prevent abuse of individuals participating in medical experiments. In addition to complex medical terms and procedures, informed consent documents also contain many concepts that are difficult for laypersons to understand, such as randomization, therapeutic misconception, equipoise, and conflict of interest [1]. While the regulations and requirements surrounding informed consent in the US are voluminous, the actual quality of informed consent document explanation is highly variable and is difficult to demonstrate or monitor [2]. This situation is exacerbated for study volunteers with inadequate health literacy - the ability to read, understand, and follow written medical instructions [3]-a classification that one third of the adults in the US fall into. As a result of these factors, there is ample evidence that a 
significant number of study participants misunderstand informed consent documents, and thus agree to participate in studies without an understanding of their commitment or the risks involved [4].

Virtual agents may provide a particularly effective solution for automatically explaining research informed consent documents to volunteers, as adjuncts to the humans administering consent. Agents can use exemplary techniques that an expert research assistant or clinician might use, given that they had training in communicating with patients with low health literacy and had unbounded time available. Virtual agents should be significantly more effective than conventional media, such as print, web, or multimedia, since face-to-face consultation with a health provider-in conjunction with written instructions - remains one of the best methods for communicating information to patients in general, but especially those with low literacy levels $[5,6]$. Face-to-face consultation is effective because it requires that the provider focus on the most salient information to be conveyed [6] and that the information be delivered in a simple, conversational speaking style. Protocols for grounding in face-to-face conversation allow providers to dynamically assess a patient's level of understanding and repeat or elaborate information as necessary [7]. Face-to-face conversation also allows providers to make their communication more explicitly interactive by asking patients to do, write, say, or show something that demonstrates their understanding [8]. Virtual agents can thus consistently evaluate patient comprehension of the information presented. Physicians infrequently evaluate patients' understanding, and when they do it is mostly simply to ask "do you understand?" without waiting for a reply [9].

In the rest of this paper we describe a series of efforts we have undertaken to automate parts of the research informed consent process using virtual agents (Figure 1). Our "baseline" agent's nonverbal behavior is synchronized with a text-to-speech engine, and user contributions to the conversation are made via a touch screen selection from a multiple choice menu of utterance options, updated at each turn of the conversation. The virtual agent has a range of nonverbal behaviors that it can use, including hand gestures, body posture shifts, gaze shifts, eyebrow raises, and head nods. Conversational nonverbal behavior is determined for each utterance using the BEAT text-to-embodied-speech system [10], with several enhancements to support health dialogues, including the ability to point at parts of the document being explained based on a model of document deictics .

\section{Related Work}

An agent that explains a document is essentially teaching the user about the topics covered in the document, and thus pedagogical strategies pioneered by other developers of virtual agents are of relevance. Virtual pedagogical agents include Autotutor [11], Persona [12], and many others.

Evaluations of these agents have largely shown mixed educational outcomes. For example, users rated the Persona agent as more entertaining and helpful than an equivalent interface without the agent [12]. However, there was no difference in actual performance (comprehension and recall of presented material) in interfaces 


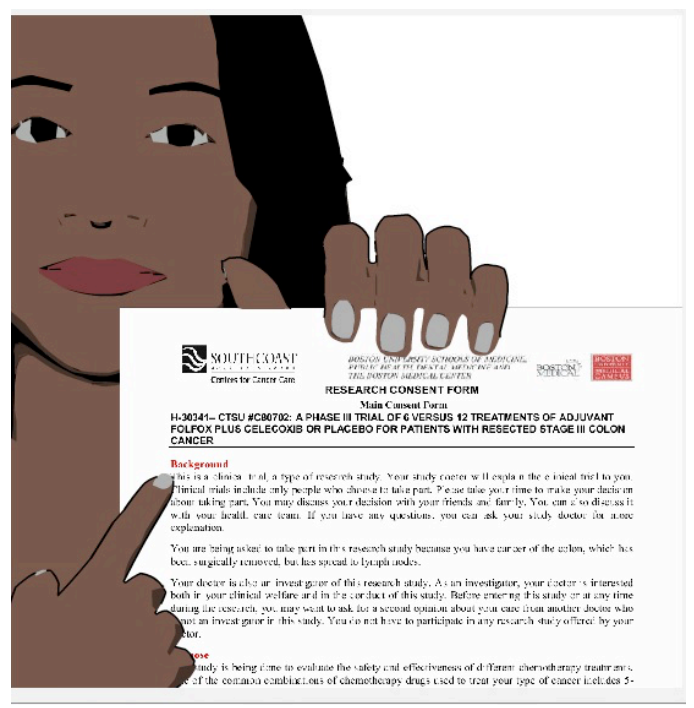

Fig. 1. Virtual Agent Explaining Informed Consent Document

with the agent vs. interfaces without it. In another study, students using the AutoTutor pedagogical agent in addition to their normal coursework outperformed both a control group (no additional intervention), and a group directed to re-read relevant material from their textbooks [13]

Bickmore, et al, reported several prior studies on virtual agents explaining medical documents to patients. In one system, a "virtual nurse" agent explained a digital copy of patients' hospital discharge instructions to them while they were still in their hospital beds $[14,15]$. Pilot and summative evaluations indicated that most patients preferred receiving their discharge instructions from the virtual nurse compared to their human doctors or nurses in the hospital, and that patients with low health literacy had significantly higher levels of satisfaction with the virtual nurse compared to patients with adequate health literacy. In another system, a virtual agent explained research informed consent documents to patients [16]. A study comparing the agent to a human research assistant and a self-study condition found that all participants were most satisfied with the consent process and were most likely to sign the consent form when the agent provided the explanation. However, the consent explanation in this system was entirely scripted for each consent document.

Fernando developed a dialogue system and virtual agent for explaining research informed consent documents that used a structured representation of each document together with a library of background concept tutorials and a few general document explanation techniques. He compared two versions of this system - a "verbose" agent that provided all relevant information about each section of a document, and a "tailored" agent that allowed users to request background information - to a selfstudy condition. He found that the verbose agent outperformed the other two conditions on comprehension test scores, but users were most satisfied with the tailored agent and least satisfied with the verbose agent [17]. 


\section{Nurse Explanation of Oncology Informed Consent Documents}

In order to inform the design of a virtual agent that administers research informed consent, we conducted a study of oncology nurses administering informed consent for clinical trials to mock study participants. In oncology clinical trials, patients will typically meet with a study nurse for an hour-long session to review the informed consent document, then take the document home to consider participation before meeting with their oncologist to answer any final questions and sign up for the trial.

We conducted detailed analyses of transcripts from several of the consent sessions. We found that the nurses structure their explanation following the structure of the informed consent document, generally proceeding linearly through the sections of the document (consistent with prior findings $[17,18]$ ). Beyond that, there was often little correlation between what the nurses said and the contents of the document, or what ethicists would say is most important information to convey (e.g., the voluntary nature of participation, potential risks, and the ramifications of randomization). The nurses spent most of their time describing what it will actually be like to experience being in the trial, reassuring the patient that they will be ok, and relating anecdotal information they happen to know that is related to information in the consent form.

Based on these findings, we proceeded to design our virtual agent for automated informed consent whose explanations followed the structure of the consent document. Informed consent documents are approved by an Institutional Review Board (IRB) ${ }^{1}$ and then cannot be changed, so any explanation offered must be entirely centered on the existing document.

\section{Evaluation of Pedagogical Strategies for Document Explanation}

As a next step in our research, we conducted a study to determine the amount of information that a virtual agent should provide to volunteers about a study, and a simple pedagogical strategy for tailoring the explanation of the informed consent document.

\subsection{Methods}

The experimental design of this study is a 3-treatment, counterbalanced, withinsubjects design, comparing three consent document explanation strategies by a virtual agent. The three strategies were, respectively, a short overview of each section only (SHORT), overview plus a detailed reading of the informed consent document (DETAILED), and an adaptive strategy in which participants were given comprehension checks of each major section of the document, and a tailored review

${ }^{1}$ The panel that reviews and approves human subjects studies at each institution to meet US federal requirements. 
based on their understanding (TAILORED). In all cases, users were given unbounded time to read each section of the digital document before proceeding.

Three informed consent documents for clinical trials involving methods for colonoscopy screening for cancer were created with different study protocols, risks, and compensation levels for use in this study. The domain of cancer screening was selected, for the documents contained a wide range of complex medical terms, facts and concepts, making it appropriate for testing an automated document explanation system. The length and complexity of the three documents were designed to be approximately the same across all three conditions.

Participants. In total, 74 subjects, $67.6 \%$ female, aged 18 to 94 years old (mean=50), participated in this study. Among all participants, 26\% had low levels of health literacy, based on the REALM screener [19].

Measures. Comprehension was assessed by a closed-book knowledge test, consisting of three YES/NO questions, and three multiple-choice questions for each document. Immediately following their interaction with the agent, participants completed a selfreport questionnaire assessing satisfaction with the consent experience, including several single-item, scale response questions, based on the Brief Informed Consent Evaluation Protocol [20] (Table 1). We created two composite measures of overall satisfaction (Q1, Q2, Q3, Q10) and attitude towards the instructor (Q1, Q3, Q4, Q5, Q6).

Table 1. Self-Report Scale Measures Completed after Agent Interaction

\begin{tabular}{|l|l|l|}
\hline Question & Anchor 1 & Anchor 7 \\
\hline Q1.How satisfied are you with the instructor? & Not at all & Very satisfied \\
\hline $\begin{array}{l}\text { Q2. How satisfied are you with the instructional } \\
\text { experience? }\end{array}$ & Not at all & Very satisfied \\
\hline $\begin{array}{l}\text { Q3. How much would you like to continue working with } \\
\text { the instructor? }\end{array}$ & Not at all & Very much \\
\hline Q4.How much do you trust the instructor? & Not at all & Very much \\
\hline Q5.How much do you like the instructor? & Not at all & Very much \\
\hline Q6. How knowledgeable was the instructor? & Not at all & $\begin{array}{l}\text { Very } \\
\text { knowledgeable }\end{array}$ \\
\hline Q7. How much information did you get? & Too little & Too much \\
\hline $\begin{array}{l}\text { Q8. How likely would you have been to sign the } \\
\text { document? }\end{array}$ & $\begin{array}{l}\text { Extremely } \\
\text { unlikely }\end{array}$ & $\begin{array}{l}\text { Extremely } \\
\text { likely }\end{array}$ \\
\hline $\begin{array}{l}\text { Q9. How much pressure did you feel to sign the } \\
\text { document? }\end{array}$ & No pressure & $\begin{array}{l}\text { Extreme } \\
\text { pressure }\end{array}$ \\
\hline Q10. How satisfied were you with the explanation? & $\begin{array}{l}\text { Extremely } \\
\text { unsatisfied }\end{array}$ & $\begin{array}{l}\text { Extremely } \\
\text { satisfied }\end{array}$ \\
\hline
\end{tabular}

\subsection{Results}

Non-parametric Friedman test were used for testing differences across the three experimental conditions for repeated measures.

Satisfaction. We found a significant effect of treatment conditions on participants' attitude towards the agent $(\mathrm{p}<.05)$, with highest satisfaction for the TAILORED 
condition, and lowest satisfaction with agent for the DETAILED condition. Similarly, we also found a significant effect of treatment conditions on participants' overall satisfaction $(\mathrm{p}<.05)$, with highest overall satisfaction for the TAILORED condition, and lowest overall satisfaction for the DETAILED condition. There was a trending effect of treatment conditions $(\mathrm{p}=.078)$ on perceived amount of information provided by the agent: participants tended to rate the SHORT and the DETAILED conditions as providing too much information compared to the TAILORED condition. No significant differences were found for likelihood to sign or perceived pressure to sign the document. Table 2 shows the descriptive statistics for the outcome measures.

Table 2. Study Results (mean and (SD))

\begin{tabular}{|l|c|c|c|c|}
\hline & SHORT & DETAILED & TAILORED & p \\
\hline Attitude Towards Agent & $5.9(1.3)$ & $5.8(1.2)$ & $6.1(1.1)$ & $<.05$ \\
\hline Overall Satisfaction & $5.7(1.3)$ & $5.4(1.4)$ & $5.9(1.2)$ & $<.05$ \\
\hline $\begin{array}{l}\text { Amount of Information } \\
\text { Provided }\end{array}$ & $5.0(1.3)$ & $5.0(1.5)$ & $4.7(1.2)$ & .078 \\
\hline Likelihood to Sign & $5.1(1.8)$ & $4.9(2.1)$ & $5.2(1.9)$ & n.s. \\
\hline Pressure to Sign & $2.3(1.8)$ & $2.5(1.9)$ & $2.2(1.7)$ & n.s. \\
\hline Comprehension & $0.92(0.7)$ & $1.04(0.8)$ & $0.93(0.8)$ & n.s. \\
\hline
\end{tabular}

Comprehension. No significant differences among treatment conditions were found for participants' total comprehension of the documents.

Health Literacy. We also investigated relationships between participants' health literacy and other measures using Spearman's rho non-parametric tests. We found that, participants' REALM scores (indicating levels of health literacy) were negatively correlated with their attitude towards the agent $($ rho $=-0.23, \mathrm{p}<.001)$, overall satisfaction (rho=- $0.15, \mathrm{p}<.05$ ), and perceived pressure to sign the document (rho=$0.15, \mathrm{p}<.05$ ), indicating that low literacy participants liked the agent more, were more satisfied with the experience, and felt more pressure to sign, compared to participants with higher levels of health literacy. Their REALM scores were also significantly correlated with their total comprehension of the consent documents (rho $=0.24$, $\mathrm{p}<.001$ ), indicating that participants with higher levels of health literacy learned more about each document.

\section{Discussion.}

In general, we found positive feedback from the participants on the consent document explanation system. Participants favored the TAILORED condition the most, rating highest on attitude towards the agent, and overall satisfaction with the experience, and they also felt the TAILORED condition provided the most appropriate amount of information compared to the other two conditions. However, we did not find any differences between approaches on comprehension. 


\section{Towards Fully-Automated Explanation}

Given that our observed human explanations of informed consent documents followed the structure of the document, and that the document must be presented to patients without modification, we further developed our virtual agent-based consent explanation system to start with an import of a consent document and support the construction of an interactive consent experience centered on the document. Our goal was to automate as much of the explanation as possible given the document contents, while allowing non-technical clinicians to provide any additional adaptations required to produce a usable consent system. Our approach is to import a structured representation of the document in XML, and drive automated explanation based on XML document annotations that could either be automatically added through analysis of the document contents, or added by a clinician via a graphical editor.

There are at least three types of annotations that can be used in marking up an informed consent document to support explanation by an agent:

Structural tags describe the structure and spatial layout of the document (e.g., SECTION, PARAGRAPH). These are essential since the agent needs to be able to display a page to the user and point at the block of text being discussed. We have used the DocBook tagset for this purpose [21].

Semantic tags describe the semantic types of content, at varying level of detail in the document (e.g., RISKS_AND_BENEFITS, RISK, STUDY_VISIT). Use of these tags assumes there is a text generator capable of providing explanatory dialogue for the tagged content.

Procedural tags serve as runtime instructions to the agent describing how it should explain some aspect of the document (e.g., SAY, COMPREHENSION_TEST, PRIORITY). These tags can be either declarative or procedural in nature, and are where most policy decisions about administration of consent for a given study site would be implemented.

Our initial approach was to tag all relevant data with semantic tags and use text generation to dynamically produce explanations. However, after reviewing over 20 sample informed consent documents we discovered that, although authors may use standard sections in their document (and many IRBs may require specific sections), there is no guarantee that authors will choose to populate these sections consistently or completely. We also observed that the authors often distribute information about a given topic across several sections of the document (e.g., there is nothing to prevent some aspects of study protocol from appearing in many different parts of a document). The definition of a hierarchical set of semantic tags (such as XML provides) presumes that all information about a given topic be contained only within a defined context, and thus is incompatible with the lack of structure we observed. In addition, following our observation of human informed consent, the agent will typically be eliding and gisting almost all of the details in the document, and offering additional information that is not represented at all in the document, thus obviating the need for semantic tags for the majority of information actually contained in the document. Finally, clinicians will require control over what the agent says about a given study in order to implement their consent policy, so some level of procedural control is required regardless. All of this led us to an annotation tag set comprised 
primarily of structural and procedural tags, which essentially instruct the agent how to explain each part of the document as it is linearly traversed by the system.

Based on this approach, we developed a set of tags that could be used to annotate an XML representation of an informed consent document, together with a library of common tutorials and other re-usable dialogue, and a visual editor that would facilitate annotation by non-technical clinicians. Most structural tags, and an initial set of procedural tags, are automatically generated by the visual editor tool when a new consent document is imported. The clinician uses the tool to iteratively extend and modify these tags and review the resulting explanation until they are satisfied with the result. Figure 2 shows the visual editor interface. The library includes descriptions of common concepts, such as voluntariness, randomization, and study overviews, that are parameterized by particular features of any given study. Clinicians can test any part of the explanation by clicking on a "preview" button.

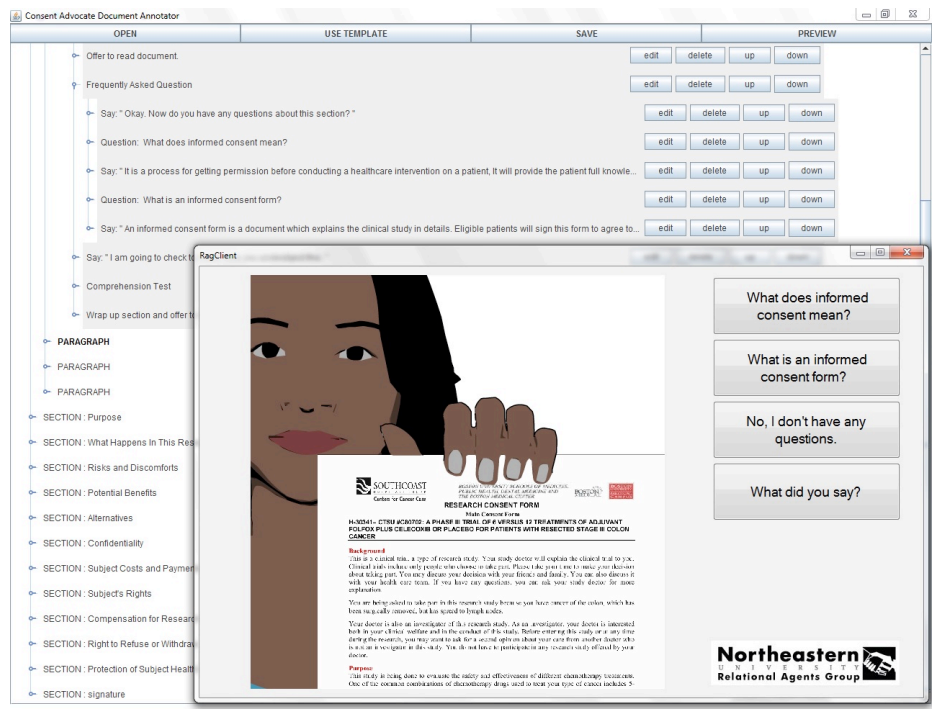

Fig. 2. Informed Consent Visual Document Annotator with Preview Running

\section{Nurse Document Tagging Usability Evaluation.}

We conducted a pilot formative evaluation on the consent annotator system at a local hospital to determine if study nurses were able to use the visual editor to annotate their consent forms. Five oncology research nurses and research associates from the hospital were recruited. Participants were first shown a demo video about how to use the system, and then received further explanation on each function. Participants were then asked to complete four simple tasks using the system. After completing the tasks, they were asked to fill out a satisfaction questionnaire (Table 3) and interviewed about their experience. In general, nurses liked the major functions the annotator system provided, such as having a dictionary to mark up complicated medical terms and the ability to add in comprehension questions. The nurses provided valuable feedback on possible improvements to the usability of the system, such as adding 
visual aids to help patients understand the documents, allowing patients to audio record their questions to the study team, and extending the system to support nonEnglish speakers.

Table 3. Nurse Ratings of Consent Document Annotator

\begin{tabular}{|c|c|c|c|}
\hline Question & \multicolumn{2}{|c|}{ Anchors } & Mean \\
\hline I found the system unnecessarily complex. & $\begin{array}{l}1=\text { Strongly } \\
\text { disagree }\end{array}$ & $\begin{array}{l}5=\text { Strongly } \\
\text { agree }\end{array}$ & $2.0(1.7)$ \\
\hline I thought the system was easy to use. & $\begin{array}{l}1=\text { Strongly } \\
\text { disagree }\end{array}$ & $\begin{array}{l}5=\text { Strongly } \\
\text { agree }\end{array}$ & $4.0(1.0)$ \\
\hline $\begin{array}{l}\text { I found the various functions in the system } \\
\text { were well integrated. }\end{array}$ & $\begin{array}{l}1=\text { Strongly } \\
\text { disagree }\end{array}$ & $\begin{array}{l}5=\text { Strongly } \\
\text { agree }\end{array}$ & $4.3(1.2)$ \\
\hline $\begin{array}{l}\text { In general, how satisfied were you with the } \\
\text { annotator? }\end{array}$ & $\begin{array}{l}1=\text { Not at all } \\
\text { satisfied }\end{array}$ & $\begin{array}{l}7=\text { Very } \\
\text { satisfied }\end{array}$ & $5.7(2.3)$ \\
\hline
\end{tabular}

\section{Conclusion}

The proper administration of informed consent is crucial to ensure the ethical treatment of human subjects, yet in practice it is poorly performed, resulting in most study participants not actually being "informed" at all. Virtual agents can greatly improve this situation, especially for individuals with low health literacy.

In this paper we have reported on a series of efforts to construct such agents, and have found that our study participants accept agents in the role of a consenting research assistant or clinician, learned well with the agent, and preferred a dynamically-tailored version of consent form explanation over a fixed presentation. We were also able to demonstrate that study nurses could use our system to develop virtual agent systems capable of explaining their own unique informed consent documents.

Our future work includes a randomized evaluation of consent agents created by study nurses in actual medical trials, comparing these agents to the standard informed consent procedure.

Acknowledgments. This work was supported, in part, by National Institutes of Health National Cancer Institute (NCI) Grant R01CA158219.

\section{References}

1. Sugarman, J., et al., Empirical research on informed consent. An annotated bibliography. Hastings Cent Rep, 1999. 29(1): p. S1-42.

2. Smith, T., E.J. Moore, and H. Tunstall-Pedoe, Review by a local medical research ethics committee of the conduct of approved research projects, by examination of patients' case notes, consent forms, and research records and by interview. Bmj, 1997. 314(7094): p. 1588-90. 
3. Ad Hoc Committee on Health Literacy for the Council on Scientific Affairs, A.M.A., Health literacy: report of the Council on Scientific Affairs. JAMA, 1999. 281(6): p. 552557.

4. Joffe, S., et al., Quality of informed consent in cancer clinical trials: a cross-sectional survey. Lancet 2001.358(9295): p. 1772-7.

5. Madden, E., Evaluation of outpatient pharmacy patient counseling. J Am Pharm Ass, 1973. 13: p. 437-443.

6. Qualls, C., J. Harris, and W. Rogers, Cognitive-Linguistic Aging: Considerations for Home Health Care Environments, in Human Factors Interventions for the Health Care of Older Adults, W. Rogers and A. Fisk, Editors. 2002, Lawrence Erlbaum: Mahwah, NJ. p. 47-67.

7. Clark, H.H. and S.E. Brennan, Grounding in Communication, in Perspectives on Socially Shared Cognition, L.B. Resnick, J.M. Levine, and S.D. Teasley, Editors. 1991, American Psychological Association: Washington. p. 127-149.

8. Doak, C., L. Doak, and J. Root, Teaching patients with low literacy skills. 2nd ed. 1996, Philadelphia, PA: JB Lippincott.

9. Schillinger, D., et al., Closing the loop: physician communication with diabetic patients who have low health literacy. Arch.Intern.Med, 2003. 163(1): p. 83-90.

10. Cassell, J., H. Vilhjálmsson, and T. Bickmore. BEAT: The Behavior Expression Animation Toolkit. in SIGGRAPH '01.2001. Los Angeles, CA.

11. Graesser, A., et al., AutoTutor: A simulation of a human tutor. Cognitive Systems Research, 1999. 1.

12. Andre, E., T. Rist, and J. Muller, Integrating reactive and scripted behaviors in a life-like presentation agent. Proceedings of AGENTS'98, 1998: p. 261-268.

13. Person, N.K., et al., Evaluating Student Learning Gains in Two Versions of AutoTutor, in Artificial intelligence in education: AI-ED in the wired and wireless future, J.D. Moore, C.L. Redfield, and W.L. Johnson, Editors. 2001, IOS Press: Amsterdam. p. 286-293.

14. Bickmore, T., L. Pfeifer, and B.W. Jack, Taking the Time to Care: Empowering Low Health Literacy Hospital Patients with Virtual Nurse Agents in Proceedings of the ACM SIGCHI Conference on Human Factors in Computing Systems (CHI). 2009: Boston, MA.

15. Zhou, S., T. Bickmore, and B. Jack, Agent-User Concordance and Satisfaction with a Virtual Hospital Discharge Nurse, in Intelligent Virtual Agents conference (IVA). 2014: Boston, MA.

16. Bickmore, T., L. Pfeifer, and M. Paasche-Orlow, Using Computer Agents to Explain Medical Documents to Patients with Low Health Literacy. Patient Education and Counseling, 2009. 75(3): p. 315-320.

17. Fernando, R., Automated Explanation of Research Informed Consent by Embodied Conversational Agents, in College of Computer and Information Science. 2009, Northeastern University: Boston.

18. Bickmore, T., L. Pfeifer, and L. Yin, The Role of Gesture in Document Explanation by Embodied Conversational Agents. International Journal of Semantic Computing, 2008. 2(1): p. 47-70.

19. Davis, T., et al., Rapid estimate of adult literacy in medicine: a shortened screening instrument. Fam Med, 1993. 25: p. 391-395.

20. Sugarman, J., et al., Evaluating the quality of informed consent. Clin Trials, 2005. 2(1): p. 34-41.

21. Walsh, N., DocBook 5: The Definitive Guide 2010: O'Reilly Associates. 